\title{
OPEN Convective meniscus splitting of polysaccharide microparticles on various surfaces
}

\begin{abstract}
Kosuke Okeyoshi ${ }^{1 凶}$, Miki Yamashita $^{1}{ }^{1}$ Kulisara Budpud $^{1}$, Gargi Joshi ${ }^{1,2}$ \& Tatsuo Kaneko ${ }^{1 凶}$
In contrast to convective self-assembly methods for colloidal crystals etc., "convective meniscus splitting method" was developed to fabricate three-dimensionally ordered polymeric structures. By controlling the geometry of evaporative interface of polymer solution, a deposited membrane with uniaxial orientation and layered structures can be prepared. Here it is demonstrated that xanthan gum polysaccharide microparticles with diameter $\sim 1 \mu \mathrm{m}$ can bridge a millimeter-scale gap to form such a membrane because the capillary force among the particles is more dominant than the gravitational force on the evaporative interface. This method is applicable for various substrates with a wide range of wettability (water contact angle, $11^{\circ}-111^{\circ}$ ), such as glass, metals, and plastics. The specific deposition can be also confirmed between frosted glasses, functional-molecules-modified glasses, and gold-sputtered substrates. By using such a universal method, the membrane formed on a polydimethylsiloxane surface using this method will provide a new strategy to design a functional polysaccharide wall in microfluidic devices, such as mass-separators.
\end{abstract}

Many strategies have been explored to fabricate colloidal crystals from microparticle dispersions based on convective self-assembly techniques developed using polymeric microspheres on planar solid substrates ${ }^{1-8}$. However, because one side of the particle is connected to or fixed on the substrates in these methods, the flexibility of the polymers is limited, restricting the use of both sides in separation membranes and stretchable materials ${ }^{9,10}$. Recently, we reported the unique phenomenon of meniscus splitting, with characteristic polymeric deposition on an evaporative air-liquid interface ${ }^{11}$. By drying an aqueous polymer solution from a cell with a 1-mm gap, the polymer deposits at specific positions with $\sim 10 \mathrm{~mm}$ intervals in the cell width direction by bridging the gap to form membranes in the depth direction. The process is strongly related to viscous fingering patterns; splitting occurs by solidification via the drying of a polymer solution ${ }^{12-18}$.

Here, we have developed a novel technique of convective meniscus splitting. Unlike the typical convective selfassembly technique, in this case, deposition occurs not only on solid substrates, but also between two air phases to form a membrane having a thickness of several tens of micrometers. Therefore, the polymeric membrane bridged the gap does not have different two sides because both sides are prepared from evaporative air-liquid interface. This technique appears applicable to various types of viscous polymer solutions, among which liquid crystalline (LC) polysaccharides were chosen to orient the self-assembled structures on the evaporative interface ${ }^{19}$. The technique has been demonstrated using several LC polysaccharides having megamolecular weights, such as sacran $\left(>10^{7} \mathrm{~g} / \mathrm{mol}\right)$ and xanthan gum $\left(X G ;>10^{6} \mathrm{~g} / \mathrm{mol}\right)^{20-22}$. XG can self-assemble as $\sim 1-\mu \mathrm{m}$-diameter microparticles dispersed stably in an aqueous solution. By drying the aqueous dispersion of XG over a millimeter-scale gap, a membrane bridging the gap is deposited having a three-dimensionally ordered structure (Fig. 1A). The distance of the cell gap strongly affects on the membrane formation and the phenomena occurs typically less than $1 \mathrm{~mm}^{20}$. This should be because of the capillary length, $\sim 2 \mathrm{~mm}$ which is an effective distance for capillary force from a plane substrate ${ }^{23}$. Furthermore, such a membrane with crosslinking points could behave as a uniaxially swellable hydrogel. These factors indicate that such polymer membranes can be assembled on many types of substrates with suitably modified preparation conditions.

In this study, meniscus splitting is examined by controlling the direction of the gravitational force and the substrate surface wettability. In our previous reports ${ }^{11,19-22}$, the demonstrated conditions for the meniscus splitting ${ }^{11}$ were limited: the water evaporation direction was parallel to the direction of the gravitational forces, and the space was sandwiched in between the non-modified smooth surface of glass substrate. However, for

${ }^{1}$ Energy and Environment Area, Graduate School of Advanced Science and Technology, Japan Advanced Institute of Science and Technology, 1-1 Asahidai, Nomi, Ishikawa 923-1292, Japan. ${ }^{2}$ Present address: B CUBE, Center for Molecular and Cellular Bioengineering, Technische Universität Dresden, Tatzberg 41, 01307 Dresden, Germany. ${ }^{\varpi}$ email: okeyoshi@jaist.ac.jp; kaneko@jaist.ac.jp 


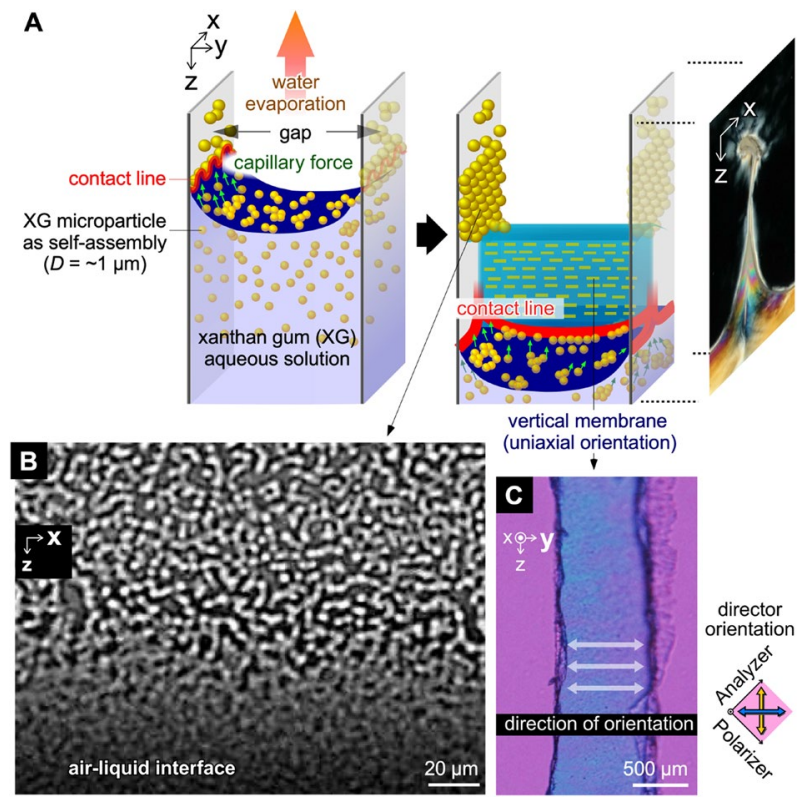

Figure 1. Deposition of polysaccharide microparticles from evaporative air-LC interface. (A) Schematic of microparticle depositions on evaporative air-LC interface and vertical membrane formation. (B) Optical microscopic images of XG deposited on a substrate. Initial concentration of XG: $0.5 \mathrm{wt} \%$. Drying at $60^{\circ} \mathrm{C}$. (C) Microscopic image of dried vertical membrane through crossed Nicols with a first-order retardation plate $(\lambda=530 \mathrm{~nm})$. Inner dimensions of the cell: (X-width, Y-thickness, Z-depth $)=(15 \mathrm{~mm}, 1 \mathrm{~mm}, \sim 20 \mathrm{~mm})$.

the development of the splitting method, it is important to clarify and expand the possibility of the conditions. Differing from previous reports ${ }^{11,19-22}$, the phenomena is demonstrated under various effects from outer environments such as the gravitational forces and the substrate surface wettability to develop the phenomena into a fixed method. Actually, meniscus splitting occurs under many conditions, i.e., it is ultimately independent of gravitational forces and substrate wettability; therefore, ordered membranes can be designed under various conditions. Near the evaporative interface, the capillary force typically acts within the effective distance from a plane substrate; it is dominant for liquids between two walls separated by a 1-mm gap rather than the gravitational force ${ }^{23}$. Furthermore, meniscus splitting is tested on various substrate materials, such as glass with/without functional-molecules-modification, metals, and plastics with a wide range of wettability, by checking the initial adsorption on these substrates.

\section{Results and discussion}

The viscous solution of XG was poured into a one-side-open cell of $15 \mathrm{~mm} \times 1 \mathrm{~mm} \times \sim 20 \mathrm{~mm}$ at $\sim 25^{\circ} \mathrm{C}$. The 1.5 -wt $\%$ aqueous solution has extremely high viscosity $\left(\sim 0.4 \mathrm{~Pa} \mathrm{~s}\right.$ at $25^{\circ} \mathrm{C}$ and $\sim 0.6 \mathrm{~Pa}$ s at $40{ }^{\circ} \mathrm{C}$ at shear rate $\left.10 \mathrm{~s}^{-1}\right)^{22}$. The sample was placed under atmospheric pressure in an oven with an air circulator and the humidity was maintained at $24 \pm 5 \%$ relative humidity $(\mathrm{RH})$ at $40{ }^{\circ} \mathrm{C}$ as measured by a hygrometer. This value is theoretically supported by an estimation from the Standard Mollier diagram in the range $13-35 \%$ RH (SI Fig. S2). By focusing on the deposited polymer, the self-assembled microparticles integrated on the substrate (Fig. 1B and Supplementary Information Fig. S1) and bridge the 1-mm-gap to form vertical membrane. The dried membrane was peeled off from the cell by mechanical widening of the cell gap and mechanical cutting from the substrates. From polarized microscopic observation with a first-order retardation plate $(\lambda=530 \mathrm{~nm})$, the membrane showed significant blue, indicating the polymer orientation in the gap direction ( $Y$-direction) (Fig. 1C, and Fig. S3). From images observed by scanning electron microscope, the microparticles with diameters of $1-2 \mu \mathrm{m}$ could be observed on the surface.

As shown in Fig. 2 and Fig. S4, the effect of gravity on the specific deposition is investigated by comparing three cases with controlled evaporation directions, using gravity parallel to the $Z$-axis, parallel to the $Y$-axis, and equal to zero. Under these conditions, the viscous solutions are retained in the cell without outflowing during drying. The XG deposition is monitored during water evaporation via crossed Nicols observation from the side to evaluate liquid crystallinity ${ }^{24,25}$. In the conditions at $25^{\circ} \mathrm{C}$ and $40^{\circ} \mathrm{C}$ (Fig. 2), the polarized images of the XG solution showed strong light transmission, indicating that the liquid crystallinity. The cross-polarized images also show slight changes in the bulk during drying, implying the low mobility of XG microparticles in the cell. Similar meniscus splitting and specific deposition are observed when the direction of gravitational force is vertical or arbitrary relative to the direction of evaporation from the cell. Thus, the effect of gravity on meniscus splitting is negligible during evaporation from the 1-mm gap. Actually, the thickness of the vertical membrane in the $X$-direction was measured by a micrometer, and it was in the range of $10-30 \mu \mathrm{m}$ in all cases. 

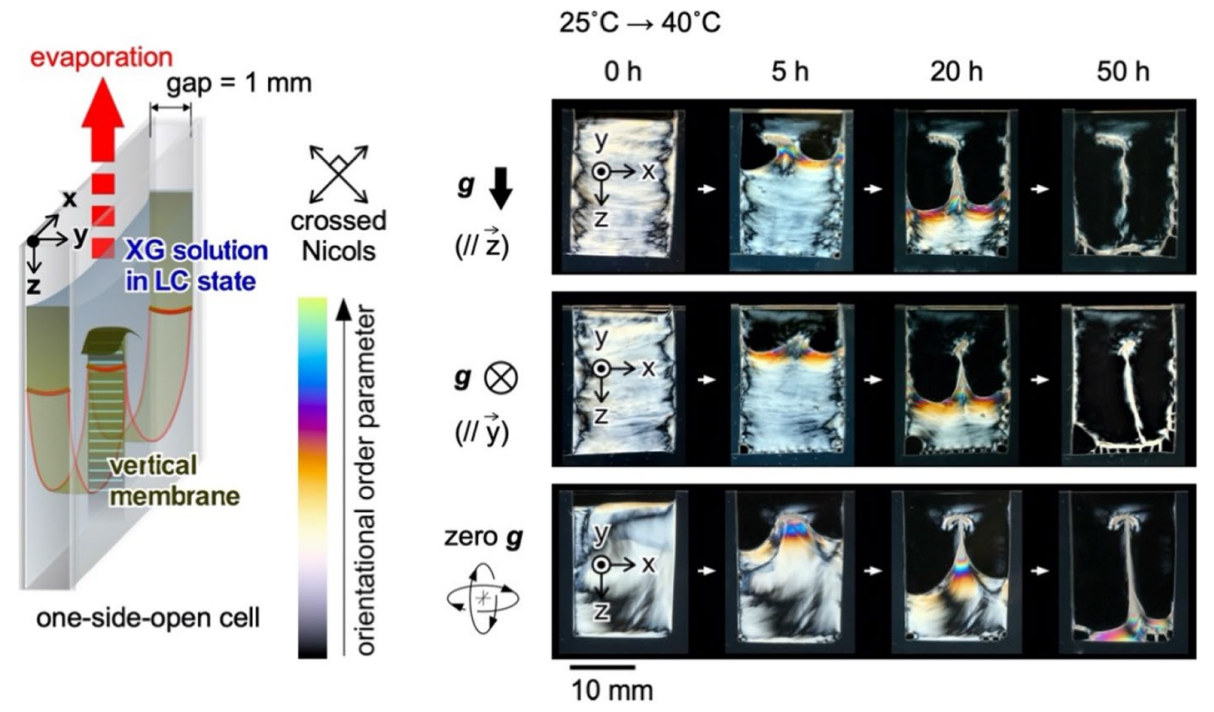

Figure 2. Effect of gravity on meniscus splitting and vertical membrane formation. Schematic of drying polymer LC solution from one-side-open cell and photographs of deposited polymer under cross-polarized light under conditions such that the gravitation force is parallel to the Z-axis, the Y-axis, and zero-gravity. Inner dimensions of the cell: (X-width, Y-thickness, Z-depth $)=(15 \mathrm{~mm}, 1 \mathrm{~mm}, \sim 20 \mathrm{~mm})$. Initial polymer concentration: $1.5 \mathrm{wt} \%$. Drying temperature: $40^{\circ} \mathrm{C}$.

Considering that the gravitational force is small, the dominant forces for this splitting should be the viscous force and the inertia force. From a view point of fluid dynamics, the initial state of the phenomena is related on plane Poiseuille flow and Reynolds number as following equations:

$$
\begin{gathered}
U(y)=\frac{1}{2 \rho v} \frac{d p}{d z}\left(y-\Delta y_{0}\right) y \\
R e \equiv \frac{U L}{v}
\end{gathered}
$$

$(U(y)$ is the flow velocity, $\rho$ is the fluid density, $v$ is the kinematic viscosity, $d p / d z$ is the pressure gradient, $L$ is the characteristic length). Because that the $U, \rho$, and $v$ are strongly dependent on time in the splitting phenomena accompanying polymer deposition, the equation should be extended by adding the spatio-temporal factors. At least, the $R e$ in the initial state is extremely small, suggesting that the viscous force is extremely larger than the inertia force: e.g., the $R e$ value is estimated to be $1.09 \times 10^{-5}$ at $40{ }^{\circ} \mathrm{C}$ for $1.5 \mathrm{wt} \%$ solution $\left(\nu=\mu / \rho=0.56 \mathrm{~m}^{2} \mathrm{~s}^{-1}\right.$, $\left.U=1.7 \mathrm{~mm} \mathrm{~h}^{-1}, L=\sim 1 \mu \mathrm{m}\right)$, and $1.01 \times 10^{-5}$ at $60^{\circ} \mathrm{C}$ for $1.0 \mathrm{wt} \%$ solution $\left(\nu=\mu / \rho=0.75 \mathrm{~m}^{2} \mathrm{~s}^{-1}, U=2.1 \mathrm{~mm} \mathrm{~h}^{-1}\right.$, $L=\sim 1 \mu \mathrm{m}$ ) on the center $y$-position of $0.5 \mathrm{~mm}$. The inertial forces are extremely smaller compared to viscous forces in these experiments, due to two primary reasons. Firstly, the xanthan solution used in the experiments has a high value of viscosity (e.g., $\sim 0.45 \mathrm{~Pa} \mathrm{~s}$ at $40^{\circ} \mathrm{C}$ at shear rate $10 \mathrm{~s}-1$ for $1.0 \mathrm{wt} \%$ xanthan solution ${ }^{21}$ ). This extremely larger viscous force induced polymeric deposition on a substrate and bridging the gap, rather than homogenization of particles dispersion during water evaporation. Additionally, the gap length of the cells, is within the capillary length that leads to formation of a barrier by condensation of microparticles at the evaporative interface. This barrier, i.e. a skin layer enhances the increase of viscous force to make a nucleus for meniscus splitting. As time passed, the Re becomes smaller because of smaller $U$ and larger $v$. The spatio-temporal evaluation is now under consideration. Due that the drastic increase of the viscosity as the polymer concentration increases $\sim 1 \mathrm{wt} \%^{21}$, time course changes of the viscosity of the fluid on the evaporative interface would be strongly related to the $R e$ value.

To clarify the effect of substrate wettability on meniscus splitting, the polymeric deposition during drying on a hydrophilic non-modified glass surface is compared with that on a hydrophobic polydimethylsiloxane (PDMS) surface (Fig. 3). In both the cases, nucleation for vertical membrane formation is observed around the center of the cell width. However, unlike the polymer adsorbed on the glass surface, that on the PDMS surface shows clearer focusing adsorption (Fig. 3A and SI Figs. S5 and S6). The thickness of the adsorbed polymer on the PDMS surface is $23 \pm 2 \mu \mathrm{m}$, much larger than that on the glass substrate $(10 \pm 2 \mu \mathrm{m})$. In case of hydrophilic surface such as glass, it showed low contact angle of polymer solution drop due to a high surface energy. This led to a good adhesion of polymer on the surface through a concave meniscus. As a result, the polymer would widely adhere on the surface as a thin film on the $X Z$-plane. In contrast, hydrophobic surface such as PDMS, it showed high contact angle due to a low surface energy. The force of cohesion between the polymer/polymer overcomes the force of adhesion between polymer/surface. This causes a poor adhesion of polymer on the hydrophobic surface. The polymer flocculated in the $Y Z$-plane to increase the interaction between polymer/polymer and to reduce the interaction between polymer/surface simultaneously. Thus, the polymer easily deposited as a thicker film in the 


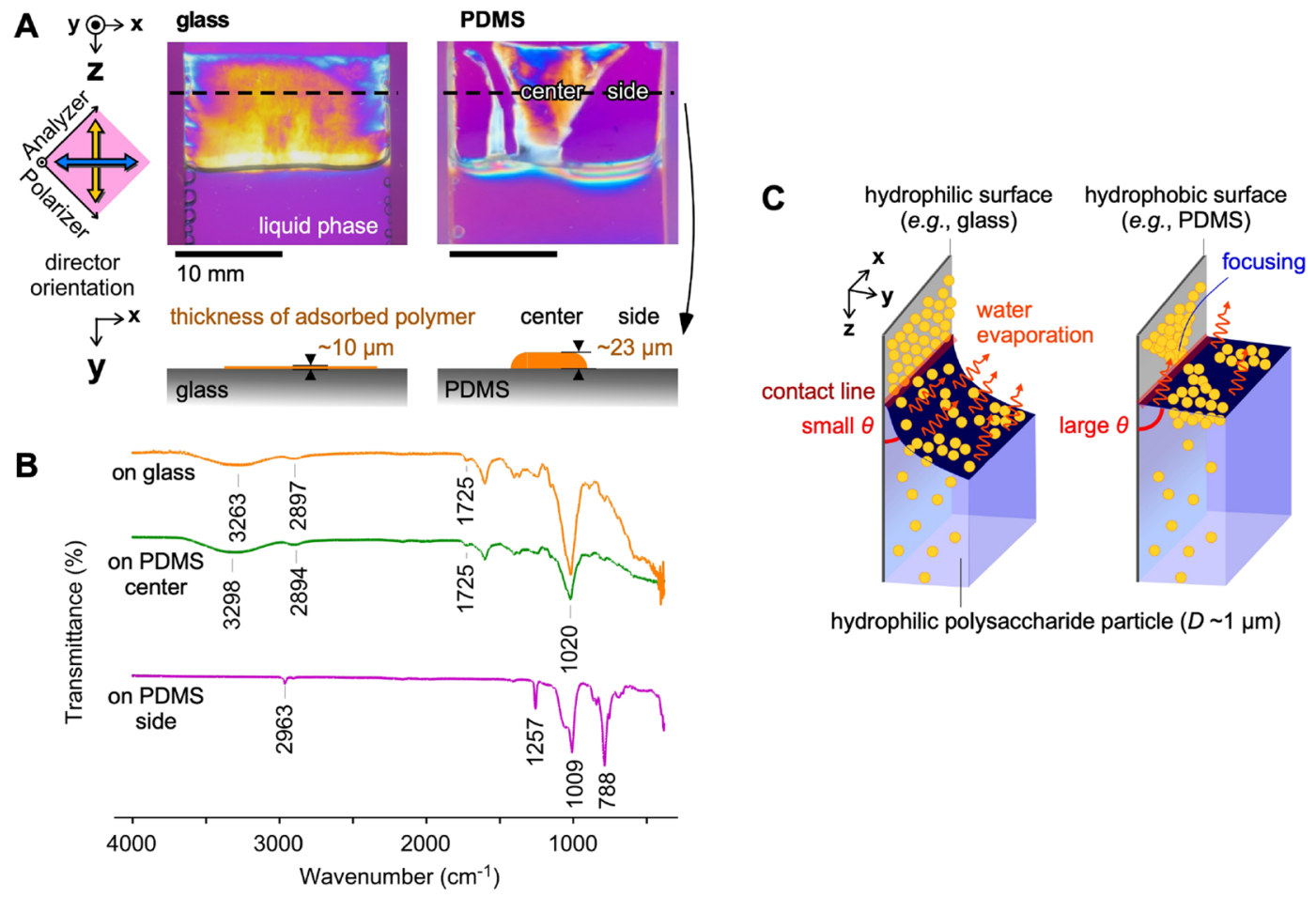

Figure 3. Evaluation of polysaccharide adsorption on glass substrates and PDMS substrates. (A) In-situ evaluation of adsorption under crossed-polarizers with a first-order retardation plate $(\lambda=530 \mathrm{~nm})$ after 8 -h drying at $60^{\circ} \mathrm{C}$. Inner dimensions of the cells: ( $X$-width, $Y$-thickness, $Z$-depth $)=(20 \mathrm{~mm}, 2 \mathrm{~mm}, \sim 20 \mathrm{~mm})$. Initial polymer concentration: $1.0 \mathrm{wt} \%$. (B) IR spectrum at given area on dotted lines. (C) Hypothesis of adsorption of polymeric particles on hydrophilic/hydrophobic surfaces by evaporative interface.

$Y$-direction on hydrophobic surface. Furthermore, the Fourier-transform infrared (FT-IR) spectra at the given positions clearly differ, as shown in Fig. 3B. First, the area on the glass surface and the center area on the PDMS surface have characteristic peaks attributed to XG at $3280 \mathrm{~cm}^{-1}$ for alcoholic O-H stretching, $2897 \mathrm{~cm}^{-1}$ for carboxy $\mathrm{O}-\mathrm{H}$ stretching, $1725 \mathrm{~cm}^{-1}$ for $\mathrm{C}=\mathrm{O}$ stretching, and $1020 \mathrm{~cm}^{-1}$ for alcoholic $\mathrm{C}-\mathrm{O}$ stretching. In contrast, the side area on the PDMS surface shows no peaks originating from XG but has characteristic peaks originating from PDMS: $2963 \mathrm{~cm}^{-1}$ for C-H stretching in $\mathrm{CH}_{3}, 1257 \mathrm{~cm}^{-1}$ for $\mathrm{CH}_{3}$ symmetric bending in $\mathrm{Si}-\mathrm{CH}_{3}, 1009 \mathrm{~cm}^{-1}$ for stretching of the siloxane bridge $\mathrm{Si}-\mathrm{O}-\mathrm{Si}$, and $788 \mathrm{~cm}^{-1}$ for $\mathrm{CH}_{3}$ rocking in $\mathrm{Si}-\mathrm{CH}_{3}$. These obvious differences indicate that the XG particles are integrated with focusing on the PDMS surface.

Such focus in deposition might be attributed to the large contact angle which provides smaller area for water evaporation (Fig. 3C). Considering that both cases have a similar evaporation rate on the whole, the water between the PDMS substrates would evaporate from smaller area and the integrated microparticle on the interface should become thicker. This situation induces a growth of thicker flocculated particles on the evaporative interface. Therefore, compared with particles on the evaporative interface between the hydrophilic surfaces, those between the hydrophobic surfaces like PDMS do not have strong adsorption on the hydrophobic surfaces. This condition induces that flocculated particle assemblies grow more easily on the evaporative interface. The growth of such flocculated assemblies creates a focusing deposition on a hydrophobic surface. Thus, the hydrophobic PDMS surface allow the hydrophilic particles to flocculate on the evaporative interface and deposit with focusing.

To apply specific deposits on different kinds of surfaces, meniscus splitting and vertical membrane formation are examined on frosted glasses (Fig. 4A). Surfaces with a wide range of roughness $(d<107 \mu \mathrm{m})$ allow the specific deposition of the polymer with vertical membrane formation because the flocculated particles on the evaporative interface are much larger than the micrometer-scale roughness. Therefore, the deposited polymers are adsorbed on the surfaces, forming bridging nuclei. On a frosted glass surface, a small increase in contact angle is obtained for water $\left(\theta_{\text {water }}: 11^{\circ} \rightarrow 17^{\circ}\right)$ with increasing surface roughness, while an extremely large increase $\left(\theta_{\mathrm{XG}}: 55^{\circ} \rightarrow 88^{\circ}\right)$ is obtained for a $1.5 \mathrm{wt} \% \mathrm{XG}$ solution. This means that the microparticles are easily integrated on the evaporative interface with a smaller interface area between the two substrates for bridging. To check membrane formation on substrates modified by functional molecules, glass substrates modified using aminopropyl triethoxysilane (APS-), Matsunami adhesive slide (MAS-), poly-L-lysine (PLL-), and silicone are used $\left(46^{\circ}<\theta_{\text {water }}<53^{\circ}\right)$ (Fig. $4 \mathrm{~B}$ ). The negatively charged XG microparticles bridge the 1-mm gaps regardless of the surface charges (such as the positive charges of the APS-, MAS-, and PLL-modified glasses; negative charges of the non-modified glasses; and the neutral charge of the silicone surface). Thus, the charges have a somewhat small effect and the microparticle interaction during deposition on the air-water interface is significantly strong. 
A

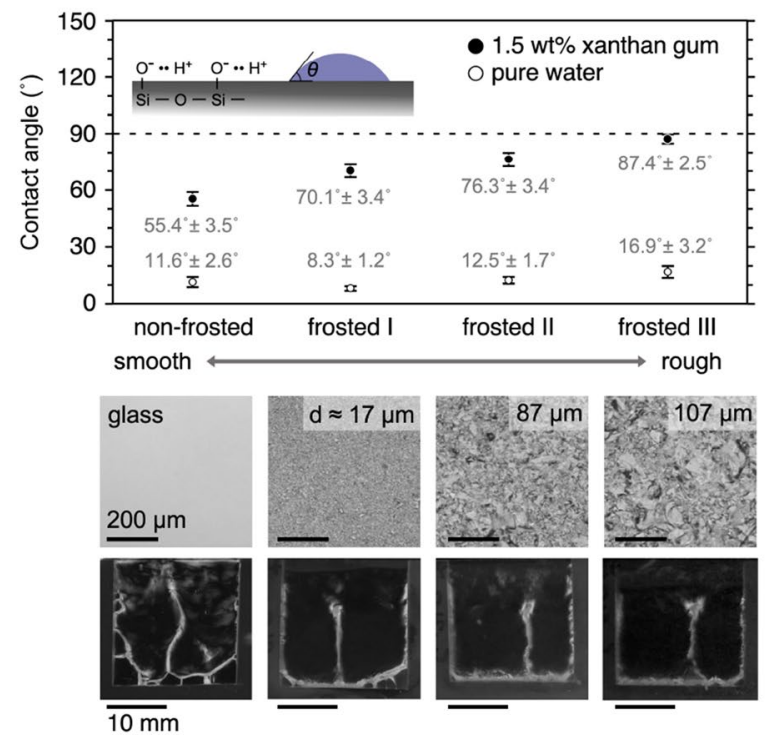

B

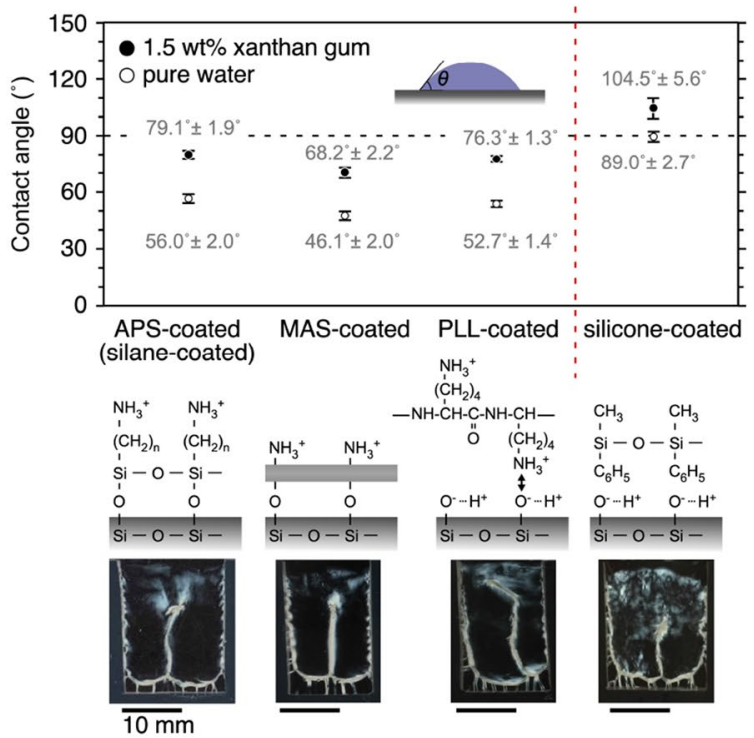

\section{C}

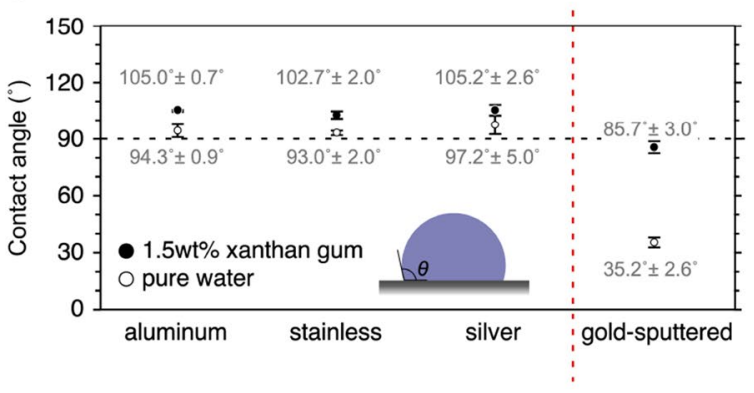

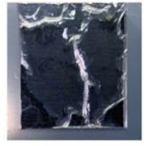

$\overline{10 \mathrm{~mm}}$
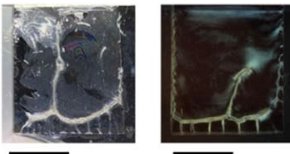

Figure 4. Macro-space partitioning on various kinds of substrates having hydrophilic/hydrophobic surfaces. Frosted glass (A), functional-molecules-modified glass (B), metals (C), and plastics (D). Contact angles on substrate for pure water and $1.5 \mathrm{wt} \% \mathrm{XG}$ aqueous solution at $25^{\circ} \mathrm{C}$. Inner dimensions of the cell: (X-width, Y-thickness, Z-depth $)=(15-20 \mathrm{~mm}, 1 \mathrm{~mm}, \sim 20 \mathrm{~mm})$. Initial polymer concentration: $1.5 \mathrm{wt} \%$. Drying temperature: $60^{\circ} \mathrm{C}$.

Deposition is also demonstrated on metals with hydrophobic surfaces $\left(\theta_{\text {water }} \approx 95 \pm 2^{\circ}\right)$ (Fig. 4 C). Similar to deposition on glass surfaces, metal surfaces like aluminum, stainless steel, and silver allowed meniscus splitting (SI Fig. S7). Such specific deposition still occurred on gold-sputtered surfaces with roughness $\left(\theta_{\text {water }} \approx 86^{\circ}\right)$. Thus, the partitioning phenomenon was observed on various metal surfaces. Similarly, deposition is studied on typical hydrophobic plastic surfaces, such as polypropylene (PP), polymethyl methacrylate (PMMA), polytetrafluoroethylene (PTFE), and PDMS (Fig. 4D). These hydrophobic substrates show large contact angles $\left(85^{\circ}<\theta_{\text {water }}<111^{\circ}\right)$, yet still allow membrane formation. Membrane formation was also confirmed on a porous polyethylene substrate with an average pore size of $17 \mu \mathrm{m}$. On rough surfaces with poor wetting $\left(\theta_{\text {water }} \approx 109^{\circ}\right)$, the deposited microparticles also successfully bridged the 1-mm gap to form vertical membranes.

\section{Conclusion}

Convective meniscus splitting was demonstrated by evaporating aqueous XG microparticle dispersions under controlled external conditions such as the direction of gravity, surface roughness, and surface wettability. Because the aqueous solution was highly viscous, the effect of direction of gravitational force was much smaller than that of the capillary force for this phenomenon. For further understanding, the correlations among the viscosity change, and the water evaporation rate would be revealed by discussing the Reynolds number in fluid dynamics in near future. We clarified that microparticles with diameters of $\sim 1 \mu \mathrm{m}$ were integrated through focusing to form 
the nucleus for bridging millimeter-scale gaps between two substrates. This demonstration would contribute the application of this phenomena in other kinds of polymers and smaller/larger size of the particle such as silica particles and microgel particles. The focusing of the microparticles occurred more strongly on hydrophobic surfaces like PDMS than on hydrophilic surfaces like glass, because the larger contact angle induced greater flocculation of microparticles on the evaporative interfaces. By using the evaporative air-LC interface, vertical membranes were successfully formed on various kinds of substrates, such as frosted glass, metals, and plastics, showing a wide range of water contact angles $\left(11^{\circ}<\theta_{\text {water }}<111^{\circ}\right)$. These observations imply that the fingering phenomenon could be applied to space-partitioning and the formation of vertical polysaccharide membranes on many kinds of substrates. By introducing crosslinking points to the membrane, it could be used in mass separators and soft actuators with directional controllability ${ }^{11,26,27}$. In the future, we envision that this technique for splitting spaces with biopolymer membranes could be applied in microfluidic devices for preparation of a membrane with uniaxial orientation capable of controlling mass-separator.

\section{Methods}

Materials. XG $\left(M_{\mathrm{w}}=4.7 \times 10^{6} \mathrm{~g} \mathrm{~mol}^{-1}\right)$ obtained from Xanthomonas campestris, neosoft XC, was purchased from Taiyo Kagaku Co. Japan. Glass substrates with a variety of surfaces were purchased from Matsunami Glass Ind. Ltd., Aluminum, stainless steel, copper (Kyuho Co., Japan), and silver (99.99\%, Inushio Precious Metals Co. Ltd., Japan) substrates were used as-purchased. Plastic substrates made of PP resin (Harue Plastic Kogeisha, Japan), PMMA resin (Harue Plastic Kogeisha, Japan), PTFE (As One Co.), and porous polyethylene film with characteristic pore size of $17 \mu \mathrm{m}$ (SUNMAP HP-5320, Nitto Denko, Co.) were used as-purchased. The PDMScoated substrates were prepared by spin-coating a mixture $(2 \mu \mathrm{L})$ of Sylgard 184 silicone elastomer base and Sylgard 184 silicone elastomer curing agent (Dow Corning Toray Co., Ltd.) on the glass substrates using a spincoater (3000 rpm, $10 \mathrm{~s})$. To fix the PDMS, it was placed in an oven at $110^{\circ} \mathrm{C}$ for $2 \mathrm{~h}$.

Drying experiments. The drying condition for the aqueous solution was set according to our previous works $^{22}$. After the XG was dissolved in pure water, the aqueous solutions were used within a few days. After removing air bubbles, the room-temperature aqueous solution was poured into a kind of one-side-open HeleShaw cell. The U-shaped cell is composed of two non-modified glass slides and glass spacers fixed with glue to prevent sample leakage. The gap distance is controlled by the thickness of the spacer. The cells were placed in an oven at constant temperature under atmospheric pressure with an air circulator (EYELA, DTC-41). Considering that the volume of the oven $(192 \mathrm{~mm} \times 270 \mathrm{~mm} \times 192 \mathrm{~mm}, \sim 10 \mathrm{~L}$ ) with the air circulator (effective exhaust velocity: $\left.\sim 40 \mathrm{~L} \mathrm{~min}^{-1}\right)$ was much larger than that of the samples $(<1 \mathrm{~mL})$, the relative humidity in the oven can be controlled by regulating the temperature. For the zero-gravity experiment, the gravity controller (Gravite, Space Bio-Laboratories, Co., Ltd.) was placed in an oven and used at $40^{\circ} \mathrm{C}$.

Observations and characterizations. To verify the degree of orientation, the samples were photographed through linear crossed-polarizers (Supporting Information, Fig. S5) ${ }^{11}$. To clarify the orientation direction, a first-order retardation plate with $\lambda=530 \mathrm{~nm}$ was put into the light path between a polarizer and the sample. Microscopic observations were made using a microscope (BX51, Olympus) equipped with a CCD camera (DP80, Olympus $)^{11}$. Fourier-transform infrared (FT-IR) spectra of the polymer adsorbed on a substrate were recorded on a spectrometer (Spectrum 100, Perkin Elmer) using a diamond-attenuated total-reflection (ATR) accessory.

Received: 20 January 2020; Accepted: 16 December 2020

Published online: 12 January 2021

\section{References}

1. Ozin, G. A., Arsenault, A. C. \& Cademartiri, L. Nanochemistry: A Chemical Approach to Nanomaterials (RSC Publishing, Cambridge, 2005).

2. Debord, J. D., Eustis, S., Debord, S. B., Lofye, M. T. \& Lyon, L. A. Color-tunable colloidal crystals from soft hydrogel nanoparticles. Adv. Mater. 16, 658 (2002).

3. Ramos, L., Lubensky, T. C., Dan, N., Nelson, P. \& Weitz, D. A. Surfactant-mediated two-dimensional crystallization of colloidal crystals. Science 286, 2325 (1999).

4. Zhang, J., Coombs, N. \& Kumacheva, E. A new approach to hybrid nanocomposite materials with periodic structures. J. Am. Chem. Soc. 12, $14512(2002)$.

5. Zhang, J., Li, Y., Zhang, X. \& Yang, B. Colloidal self-assembly meets nanofabrication: From two-dimensional colloidal crystals to nanostructure arrays. Adv. Mater. 22, 4249 (2010).

6. Grzelczak, M., Vermant, J., Furst, E. M. \& Liz-Marzan, L. M. Directed self-assembly of nanoparticles. ACS Nano 4, 3591 (2010).

7. Hatton, B., Mishchenko, L., Davis, S., Sandhage, K. H. \& Aizenberg, J. Assembly of large-area, highly ordered, crack-free inverse opal films. Proc. Natl. Acad. Sci. USA 107, 10354 (2010).

8. Vogel, N., Weiss, C. K. \& Landfester, K. From soft to hard: The generation of functional and complex colloidal monolayers for nanolithography. Soft Matter 8, 4044 (2012).

9. Pendergast, M. M. \& Hoek, E. M. V. A review of water treatment membrane nanotechnologies. Energy Environ. Sci. 4, 1946 (2011).

10. Sekitani, T. et al. A rubberlike stretchable active matrix using elastic conductors. Science 321, 1468 (2008).

11. Okeyoshi, K., Okajima, M. K. \& Kaneko, T. Emergence of polysaccharide membrane walls through macro-space partitioning via interfacial instability. Sci. Rep. 7, 5615 (2017).

12. Saffman, P. G. \& Taylor, G. The penetration of a fluid into a medium or Hele-Shaw cell containing a more viscous liquid. Proc. $R$. Soc. Lond. Ser. A. 245, 312 (1958). 
13. Cazabat, A. M., Heslot, F., Troian, S. M. \& Carles, P. Fingering instability of thin spreading films driven by temperature gradients. Nature 346, 824 (1990).

14. Brzoska, J. B., Brochard-Wyard, F. \& Rondelez, F. Exponential growth of fingering instabilities of spreading films under horizontal thermal gradients. Europhys. Lett. 19, 97 (1992).

15. Lindner, A., Coussot, P. \& Bonn, D. Viscous fingering in a yield stress fluid. Phys. Rev. Lett. 85, 314 (2000).

16. Makino, K., Kawaguchi, M., Aoyama, K. \& Kato, T. Transition of viscous fingering patterns in polymer solutions. Phys. Fluids 7, 455 (1995).

17. Nagatsu, Y., Matsuda, K., Kato, Y. \& Tada, Y. Experimental study on miscible viscous fingering involving viscosity changes induced by variations in chemical species concentrations due to chemical reactions. J. Fluid Mech. 571, 475 (2007).

18. Rabbani, H. S. et al. Suppressing viscous fingering in structured porous media. Proc. Natl. Acad. Sci. USA 115, 4833 (2018).

19. Okeyoshi, K., Okajima, M. K. \& Kaneko, T. Milliscale self-integration of megamolecule biopolymers on a drying gas-aqueous liquid crystalline interface. Biomacromol 17, 2096 (2016).

20. Okeyoshi, K., Joshi, G., Okajima, M. K. \& Kaneko, T. Formation of polysaccharide membranes by splitting of evaporative air-LC interface. Adv. Mater. Inter. 5, 1701219 (2018).

21. Joshi, G., Okeyoshi, K., Mitsumata, T. \& Kaneko, T. J. Micro-deposition control of polysaccharides on evaporative air-LC interface to design quickly swelling hydrogels. Colloid Interf. Sci. 546, 184 (2019).

22. Okeyoshi, K. et al. Effect of evaporation rate on meniscus splitting with formation of vertical polysaccharide membranes. Adv. Mater. Inter. 6, 1900855 (2019).

23. de Gennes, P. G., Brochard-Wyart, F. \& Quere, D. Capillarity and wetting phenomena: Drops, bubbles, pearls, waves. Springer https ://doi.org/10.1007/978-0-387-21656-0 (2003).

24. Takada, Y., Sato, T. \& Teramoto, A. Dynamics of stiff-chain polymers in isotropic solution. 2. Viscosity of aqueous solutions of xanthan, a rigid double-helical polysaccharide. Macromolecules 24, 6215 (1991).

25. Rwei, S. P. \& Nguyen, T. A. Liquid crystalline phase in xanthan gum (XG)/H 2 O/H 3 PO 3 and XG/H 2 O/H 3 PO 4 tertiary systems: A thermal and rheological study. Cellulose 21, 3231 (2014).

26. Okajima, M. K. et al. Anisotropic swelling in hydrogels formed by cooperatively aligned megamolecules. RSC Adv. 5, 86723 (2015).

27. Joshi, G., Okeyoshi, K., Okajima, M. K. \& Kaneko, T. Directional control of diffusion and swelling in megamolecular polysaccharide hydrogels. Soft Matter 12, 5515 (2016).

\section{Acknowledgements}

This work was supported by Grant in Aid for Scientific Research on Innovative Area (JP20H05213) from the Ministry of Education, Culture, Sports, Science, and Technology of Japan, The Asahi Glass Foundation, The Innovation Inspired by Nature Research Support Program, Sekisui Chemical Co. Ltd, and Shibuya Science Culture and Sports Foundation. G. J. is grateful for the research fellowships of the Japan Society for the Promotion of Science for Young Scientists (JP18J11881).

\section{Author contributions}

K.O. wrote the manuscript. K.O., M.Y., K.B. and G.J. carried out the experiments. K.O. and T.K. supervised the experiments. All authors reviewed the manuscript.

\section{Competing interests}

The authors declare no competing interests.

\section{Additional information}

Supplementary Information The online version contains supplementary material available at https://doi. org/10.1038/s41598-020-80779-Z.

Correspondence and requests for materials should be addressed to K.O. or T.K.

Reprints and permissions information is available at www.nature.com/reprints.

Publisher's note Springer Nature remains neutral with regard to jurisdictional claims in published maps and institutional affiliations.

(c) (i) Open Access This article is licensed under a Creative Commons Attribution 4.0 International License, which permits use, sharing, adaptation, distribution and reproduction in any medium or format, as long as you give appropriate credit to the original author(s) and the source, provide a link to the Creative Commons licence, and indicate if changes were made. The images or other third party material in this article are included in the article's Creative Commons licence, unless indicated otherwise in a credit line to the material. If material is not included in the article's Creative Commons licence and your intended use is not permitted by statutory regulation or exceeds the permitted use, you will need to obtain permission directly from the copyright holder. To view a copy of this licence, visit http://creativecommons.org/licenses/by/4.0/.

(C) The Author(s) 2021 